\title{
Current Therapy
}

National Cancer Institute

\section{Source}

National Cancer Institute. Current Therapy. NCI Thesaurus. Code C85582.

The name of the therapy that is currently being administered. 